\title{
Turning Visitors into Customers: A Usability-Centric Perspective on Purchase Behavior in Electronic Channels
}

\author{
Viswanath Venkatesh \\ Sam M. Walton College of Business, University of Arkansas, Business Building, Fayetteville, Arkansas 72701, \\ vvenkatesh@vvenkatesh.us \\ Ritu Agarwal \\ Robert H. Smith School of Business, University of Maryland, Van Munching Hall, College Park, Maryland 20742-1815, \\ ragarwal@rhsmith.umd.edu
}

\begin{abstract}
W e develop a theoretical model for predicting purchase behavior in electronic channels. The model suggests that website use (i.e., technology use), a key indicator of the degree to which a site is "sticky," is a significant antecedent of purchase behavior. Furthermore, we relate the usability of a website to use behavior and purchase behavior. Specifically, individual characteristics and product type are argued to differentially influence the weights that customers place on five different categories of usability. The weighted ratings of the five categories together determine use behavior and purchase behavior, after controlling for purchase need, experience with similar sites, and previous purchase on the specific sites. The model was tested in a longitudinal field study among 757 customers who provided usability assessments for multiple websites from four different industries-i.e., airlines, online bookstores, automobile manufacturers, and car rental agencies. Six months later, 370 of these individuals provided responses to help understand the transition from visitor to customer, i.e., whether they actually transacted with a specific website. Results provided strong support for the model and yield important theoretical and practical implications.
\end{abstract}

Key words: usability; online consumer behavior; website use; online purchase

History: Accepted by Rajiv D. Banker, information systems; received July 8, 2003. This paper was with the authors $10 \frac{1}{2}$ months for 2 revisions.

\section{Introduction}

Most e-commerce sites rebuff at least $70 \%$ of the customers who visit them, passing up millions of dollars in potential sales. Even the best retail Web sites are doing only half the business they could be doing, researchers say. The culprit: poor Web site usability. (Lais 2002, p. 44)

Electronic channels are an important avenue for firms to reach their customers and to generate sales (Moe and Fader 2004). Indeed, the value proposition of electronic commerce is that it can aid firms in transcending physical barriers to reaching customers and increase the size of the customer base. However, this reach is predicated on the assumption that customers will use the firm's electronic channels-specifically, its website-to engage in commerce. Although there is a significant body of work related to individual behavior in the context of new technologies (see Venkatesh et al. 2003 for a review), websites constructed for customers pose at least two challenges for firms not widely studied in prior information systems (IS) research on technology use. First, consumers are typically outside the immediate sphere of managerial influence, thereby limiting the potential for interventions to promote adoption and use. Second, because websites exhibit significant variety in capabilities and functionality, specifying the requirements for such systems is complex. Recent statistics indicate that although there is a growing number of individuals using the Internet to perform product research, price comparisons, and the like, the number of actual purchases made online is still relatively small (Moe and Fader 2004). Thus, a concern for organizations is to understand the barriers and facilitators of online purchasing and to construct websites that can turn visitors into paying customers.

Prior research examining online purchase behavior has approached the problem from several diverse perspectives. Using technology acceptance theories, there is evidence that individual beliefs such as usefulness and ease of use predict the extent to which customers will buy online (Chen et al. 2002). Others have posited and empirically confirmed that consumer attitudes toward online shopping and intentions to shop online are influenced by product perceptions, 
the attributes of the shopping experience, customer service, and consumer risk (Jarvenpaa and Todd 1996/1997). Additionally, the effects of various demographic characteristics such as income, education, Internet use, Internet search, and perceptions of Web vendors' sales processes on retail purchasing behavior have been studied (Burroughs and Sabherwal 2002). In an attempt to better understand online consumer behavior, scholars have developed segmentation models of Internet shoppers (e.g., Burke 2002, Moe 2003). Finally, some researchers have examined the relationship between specific design elements of a virtual store and important outcomes such as sales volume (e.g., Lohse and Spiller 1999).

To further our understanding of website use and online purchase behavior, we use an alternative theoretical lens-i.e., usability-to predict purchase behavior in the context of business-to-consumer (B2C) electronic commerce. As defined by the International Standards Organization (ISO), usability is the "extent to which a product can be used by specified users to achieve specified goals with effectiveness, efficiency, and satisfaction in a specified context of use" (Karat 1997, p. 34). The importance of usability in the study of online behaviors has been noted in human-computer interaction (HCI) and IS research. $\mathrm{HCI}$ researchers have argued that the use of a website is influenced by its usability (e.g., Nielsen 2000). Both HCI and IS researchers observe that usability is a key metric for assessing the quality of a range of different systems, including websites (e.g., Agarwal and Venkatesh 2002, Palmer 2002, Nielsen 2000). Because usability is associated with important outcomes such as error reduction, positive attitudes, and increased use (Lecerof and Paterno 1998, Nielsen 2000), we expect it to be an important determinant of online purchase behavior. Our conceptualization of usability (see Agarwal and Venkatesh 2002) is rich and complements and extends prior work in HCI and IS.

Although the importance of website usability has been acknowledged, there has been limited research examining the effects of usability on purchase behavior. Arguably, consumer purchase behavior is of significance not only to marketing researchers and practitioners, but also to organizations seeking to understand the impact of their Web presence on commerce. We pose the following question: What influences consumer perceptions of website usability, and does the usability of a website have an effect on subsequent purchases from that website? To answer this question, we develop a nomological net for usability that encompasses both its determinants and consequences. To identify determinants of usability, we draw upon research in consumer behavior that has investigated online shopping environments. In predicting purchase behavior, in addition to usability, we employ website use (i.e., technology-use behavior) as a key predictor. Use behavior has been a central dependent variable in much IS research, particularly in the domain of user adoption, and is one of the key system success constructs (see DeLone and McLean 1992, Seddon 1997). In the context of websites, use behavior represents the degree to which a site is "sticky," in that it attracts users often and retains them for longer periods of time (Rosen 2001). We tested the proposed model via secondary analysis of data reported in Agarwal and Venkatesh (2002), where 757 users provide usability assessment for multiple websites. The prior data, additional data gathered at the same time and not reported earlier, and new data gathered from a subset of users $(N=370)$ six months later about their use and purchase behavior on these sites yields a longitudinal panel for model testing.

\section{Theory}

The overall nomological net for the theorized determinants and consequences of website usability is shown in Figure 1. We present the theoretical background for usability that builds on research in HCI and IS. Next, we draw upon theories in IS, psychology, consumer information processing, and consumer behavior to develop specific hypotheses related to the determinants and consequences of website usability.

\section{Background: Usability and Its Categories}

Usability has had a pivotal role in decades of $\mathrm{HCI}$ research, where a focal tenet is that in order for technological devices to be successful, their design must pay attention to the complexities associated with the human-computer interface (e.g., Shneiderman 1980). As observed recently by Olson and Olson (2003), "HCI is the study of how people interact with computing technology" (p. 492). The goal of HCI research, then, is to understand how to design and engineer more usable artifacts. Guidelines, techniques, and methods emerging from $\mathrm{HCI}$ research have been used to inform the design of personal digital assistants (Milewski and Smith 2000), investigate errors made while utilizing alternative modeling approaches (Agarwal et al. 1999), and examine usability of virtual environments (Barfield and Furness 1997).

In the context of websites, usability has gained prominence based on the influential work of Nielsen (2000). He asserts that usability engineering is key to commercial website design and argued that an organization that does not pay attention to this aspect is not likely to generate traffic to its website or, even if visitors stop by, the site will lack the crucial attribute of "stickiness." Researchers also acknowledge that usability cannot be conceptualized independently of the context in which it is to be assessed: Lecerof and Paterno (1998) argue that what constitutes usability is 
Figure 1 Theoretical Model

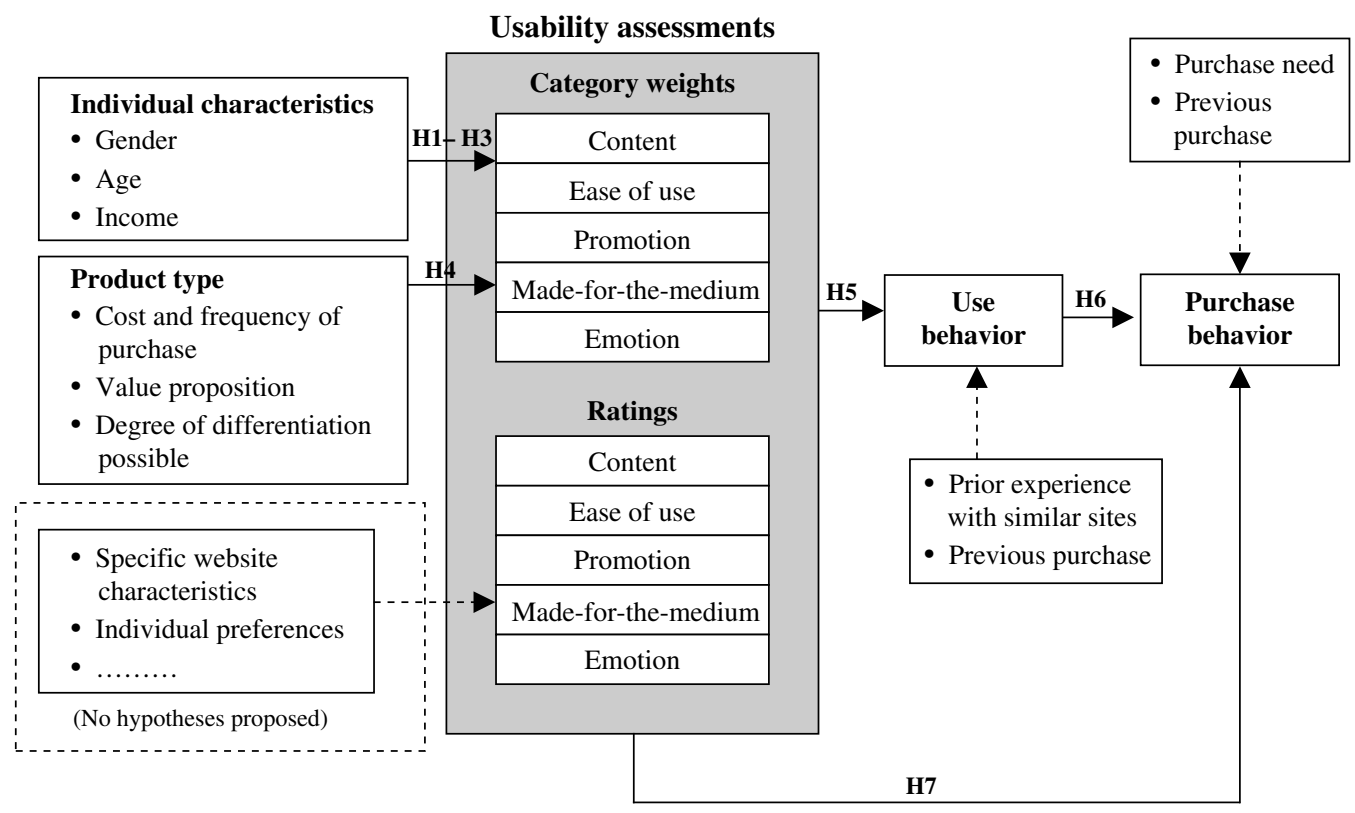

contingent upon both the task for which the system is to be used as well as the target users. For example, fun may be a primary criterion for systems designed for use by children, while efficiency is likely to be a major usability goal in the design of banking systems. Prior research has proposed multiple factors by which the usability of websites may be assessed. Eighmey and McCord (1998) derived 17 factors tapping into different aspects of website usability that were subsequently reduced to nine groups, including dimensions such as personal involvement, useful information, simplicity of organization, and desire for relationship. Likewise, Nielsen (2000) offers a wide range of design principles for a usable system, derived from a synthesis of extensive prior work.

The specific aspects of usability examined here are those incorporated into the usability metric and instrument described in Agarwal and Venkatesh (2002). The metric and instrument uses Microsoft's Usability Guidelines (MUG), a fairly comprehensive collection of available usability criteria that includes a broad range of attributes relevant to consumer evaluations of websites (Agarwal and Venkatesh 2002). The guidelines include five major categories that collectively tap into different aspects of website usability: content, ease of use, promotion, made-for-the-medium, and emotion. Four of the five categories (i.e., except promotion) are further decomposed into subcategories that specify finer-grained usability attributes. The categories and subcategories that comprise the usability metric are consistent with the ISO definition of usability. Particularly, the dimensions of effectiveness, efficiency, and satisfaction emphasized in the
ISO definition relate to MUG categories in the following way: (a) content and made-for-the-medium tie to effectiveness goals; (b) ease of use and made-for-themedium tie to efficiency goals; and (c) promotion and emotion tie to satisfaction. Additionally, the MUG categories have been shown to possess content validity (see Agarwal and Venkatesh 2002 for a development of the metric).

It is also noteworthy that some MUG categories overlap with factors that have been shown to shape individual behaviors towards information technologies in prior IS literature. Particularly, "content" is similar to perceived usefulness, or the instrumental outcomes associated with technology use in a variety of technology adoption models; similarly, ease of use, tapping into the cognitive effort required to use an artifact, recurs as an important driver of technology-use intentions in several models of adoption (Agarwal and Prasad 1998, Taylor and Todd 1995, Venkatesh et al. 2003). Likewise, "emotion" reflects affective reactions to websites-such reactions have been shown to be important to a variety of technology-use behaviors (Agarwal and Karahanna 2000, Venkatesh 2000).

While demonstrating some commonality with prior IS research on technology acceptance, the MUG categories considerably extend the range of attributes being examined. For instance, content is subdivided into relevance, media use, breadth and depth, and current and timely information. Thus, unlike perceived usefulness, which assesses the degree to which using an information system improves an individual's performance, content offers a more detailed and granular assessment; the same is also true of ease of use 
and emotion. Additionally, MUG includes categories of made-for-the-medium and promotion, which are not featured in extant models of technology acceptance (see Venkatesh et al. 2003) but are nonetheless critical to the evaluation of websites. Therefore, the MUG categories provide a robust and detailed basis for assessing website usability. Empirically, Agarwal and Venkatesh (2002) demonstrated that usability assessments as measured by MUG correlate very highly with overall usability assessments of websites, thereby providing support for the validity of the MUG metric and instrument.

\section{Hypothesis Development}

In their review of $\mathrm{HCI}$ research of the past three decades, Olson and Olson (2003) observe that HCI researchers are increasingly interested in modeling context-specific, as opposed to generic, behaviors with technological artifacts. Such context dependence has been alluded to in the domain of consumer behavior, as well as where researchers acknowledge that evaluative criteria differ in type, number, and importance (Hawkins et al. 1995); and much like consumer behavior in general, criteria are dependent on the consumer, the product, and the situation (Belk et al. 1989). For example, simple products tend to be evaluated using a limited set of factors, while an expensive product is likely to entail the assessment of many more evaluative criteria prior to a positive purchase decision. In addition to the criteria themselves being influenced by the consumer, the product, and the situation, there is evidence to suggest that the relative importance assigned to each criterion differs systematically across multiple consumption situations characterized by different configurations of products and consumers. Thus, marketers are concerned not only with identifying the specific evaluative criteria that consumers are likely to use, but also with the relative importance (weights) each individual consumer assigns to these criteria. Such information is also critical for IS designers because it helps isolate specific features that different types of individuals in different situations would desire in a technological artifact. Given this background, we examine the drivers of the importance (weights) of usability categories next.

Individual and Product Characteristics. A substantial body of research in IS and marketing theorizes individual characteristics, particularly demographic variables, as predictors of technologyrelated behaviors (Venkatesh et al. 2003) and consumer behaviors (Morton et al. 2001, Vakratsas 1998). The three demographic variables that are consistently related to technology perception, technology use, and/or consumer behavior are gender, age, and income. Consumer behavior research also suggests that the nature of the product plays an important role in consumer information processing and search in both online and offline environments (Chiang and Dholakia 2003). Particularly, demographic variables have been utilized extensively not only to explain and predict consumer behavior, but also to segment consumers more effectively with regard to their purchase propensities, such that more homogeneous behaviors are expected within groups and more heterogeneous across (Dawar et al. 1992). On the basis of research in IS and consumer behavior in general, and consumer information processing in particular (Burke 2002), we argue that individual characteristics and product characteristics will influence what consumers deem to be important in the design of a website, i.e., the weights they assign to different usability categories. However, because affective reactions to specific aspects of usability, i.e., ratings, are likely to be driven by factors such as the specific design choices made for a particular website, and preferences such as whether individuals prefer tabular or graphical displays, there is no ex ante expectation that individual or product characteristics examined here will be causally related to ratings. As shown in Figure 1, although we acknowledge the existence of these other factors, they are outside the scope of this current research.

Gender. Among the traditional demographic variables, gender plays a crucial role in consumption evaluation, with women and men exhibiting different behaviors toward a multitude of product types (Barletta 2003, Dawar et al. 1992). Examples of these different behaviors are available in research that shows that women interpret advertising messages differently than men (Prakash 1992), men have distinct preferences regarding durable goods such as automobiles (Triplett 1994), and men constitute a significant group of consumers of leisure and sports products (Zinn 1991). Gender plays a role in the evaluation of service encounters-while women tend to focus on the relational aspects of the encounter, men pay greater attention to the substantive aspects of the service and outcomes achieved (Barletta 2003). Gender differences arising from differential relational needs persist in the context of behaviors on the Internet as well—as observed by Smith and Whitlark (2001), although men have stronger preferences for information, women tend to prefer community-building activities and are more responsive to emotional appeal. Smith and Whitlark further point out that the functional needs of men and women for websites are likely to vary.

With regard to computing technology in general and Web-based shopping in particular, gender has been shown to be an important predictor of attitudes (Burke 2002, Van Slyke et al. 2002). Specifically, there is evidence to suggest that men would place 
greater weight on usefulness perceptions, whereas women would place greater weight on ease-of-use perceptions (Venkatesh and Morris 2000). As noted earlier, there is similarity between those two constructs and content and ease-of-use categories, respectively, and therefore this will likely manifest as a greater weight being assigned by men to content and women to ease of use. Further, women's greater emphasis on relational aspects is supported by evidence that women are more influenced by social norms when making decisions about technology adoption (Venkatesh and Morris 2000). This, combined with women's preference for emotional appeal, is likely to be exhibited in Web contexts via a greater emphasis on promotion and emotion by women. Because gender differences in made-for-the-medium can be argued both from the perspective of enhancing effectiveness (content) and efficiency (ease of use), we expect the weights to be comparable across women and men. Specifically, the community component is likely to be more important to women because of their interest in relationship building. However, the personalization component can help enhance access to content (effectiveness) and help do so with less effort (efficiency), thus appealing to both women and men. The potential for refinement and addition of content will likely be more important to men because of the ties to usefulness. Therefore, we hypothesize:

Нyротнеsis 1. Gender will influence the weights individuals assign to the usability categories such that (a) content will be more important to men; (b) ease of use, promotion, and emotion will be more important to women; (c) made-for-the-medium will be equally important to women and men.

Age. Age has a significant influence on consumer evaluation of products, with older consumers displaying preferences that are different (Moschis 1991) from those of younger consumers (Fay 1993). In behaviors on the Internet in particular, age has been found to be strongly related to adoption and use (Feller 2003). There are different theoretical pathways through which the effects of age on the importance of usability categories can be explained. To the extent that younger consumers are more socialized with technology, they are likely to place less importance on ease of use, which is empirically confirmed (see Venkatesh et al. 2003). Likewise, we expect older consumers to be typically more thoughtful and deliberate in their evaluation processes and, therefore, assign higher weight to content (Burke 2002). As the most avid consumers of websites, younger visitors are likely to value the ability to connect electronically with others and, arising from their need to express unique self-identities (Bao and Shao 2002), they will tend to have a stronger preference for sites that treat them in a personalized fashion. Both community and personalization are aspects of made-for-the-medium category, thus making this category more important to younger consumers. However, we find no plausible mechanism whereby there would be difference between older and younger consumers with regard to the importance of promotion or emotion. Therefore, we hypothesize as follows.

Hypothesis 2. Age will influence the weights individuals assign to the usability categories such that (a) content and ease of use will be more important to older consumers; (b) made-for-the-medium will be more important to younger consumers; (c) promotion and emotion will be equally important to younger and older consumers.

Income. Income is significant because it drives consumer information processing and search behaviors. Household income affects consumption choices by virtue of the purchasing power and status it confers, and has been shown to have an impact on consumption in several product categories (Simmons Market Research Bureau 1991). To the extent that income affects consumption choices and expectations, we expect it to influence the importance consumers assign to the various categories of website usability. Higher-income individuals have higher opportunity costs for their time, and desire to complete transactions as quickly and efficiently as possible (Pashigian et al. 2003). Such time-convenience aspects have been shown to be significantly related to consumer decisions to shop online (Chiang and Dholakia 2003) and therefore, higher-income consumers would likely value content and ease of use substantially. Similarly, to the degree that customization offers greater efficiency in search (Diehl et al. 2003), reduces cognitive costs, and delivers valuable content (Haubl and Trifts 2000), the ability of a website to offer a personalized experience will be more important to higher-income consumers. This importance of personalization will be reflected in the higher importance of made-for-the-medium to higher-income consumers. Higher-income consumers seek to reassure themselves about product quality and because advertising is typically viewed as a signal of product quality (Lynch and Ariely 2000, Milgrom and Roberts 1986) and, therefore, will place greater importance on promotion. However, there is no theoretical or empirical evidence supporting a relationship between income and the category of emotion. Therefore, we hypothesize the following.

Hypothesis 3. Income will influence the weights individuals assign to the usability categories such that (a) content, ease of use, promotion, and made-for-the-medium will be more important to high-income consumers; (b) emotion will be equally important to low- and high-income consumers. 
Product Type. We theorize that the nature of the product has an effect on what consumers seek from a website that offers the product. Recently Peterson et al. (1997) noted that it is important to incorporate product and service characteristics into discussions of the use of the Internet for marketing. They argue that the traditional dichotomy of products and services as search goods versus experience goods needs to be revisited in light of the capabilities of the Internet. Whereas search goods are characterized by product attributes that are fully known prior to purchase, experience goods are those where either information search is costly, or product attributes cannot be reliably determined prior to purchase and trial. Peterson et al. (1997) proposed an alternative classification that is posited to be more relevant for Internet marketing where goods are distinguished along three dimensions: (1) cost and frequency of purchase; (2) value proposition, i.e., whether the good is tangible and physical or intangible and service related; and (3) degree of differentiation possible. They further suggested that consumer decision processes and search behaviors are likely to vary in accordance with specific constellations of these three dimensions. With this backdrop, we expect user evaluations of websites to be influenced by the type of product the website is designed to support.

Additional support for the influence of product type on consumer search behavior in an online setting is provided by Hahn and Kauffman (2002). Using information-foraging theory as the conceptual framework, they classify products into three categories: convenience goods, researched goods, and replenishment goods. Each product category is differentiated on the basis of the consumer behavior dimensions of need arousal, information search, product evaluation, purchase decision, and postpurchase evaluation. They then argue that because consumer behavior is different across the product types, the specific capabilities and features of the website required to support such behaviors are likely to vary across product categories.

Building upon the theoretical arguments presented in prior work, we suggest that the importance of usability categories will be systematically related to product types. There are a variety of ways in which product type can influence the relative importance of usability categories for consumers and, given the multiple classifications of product types in the literature as well as the multiple dimensions along which products can be differentiated, our objective is not to develop specific hypotheses relating different product types to usability categories. Rather, we seek to explore the nature of the influence of products on the weights assigned to different usability categories.

What are the usability categories that are likely to be assigned different weights across different product types? Because shopping is an inherently informationintensive activity that involves search, evaluation, and selection, the importance of content will be uniformly high across all product categories (e.g., Bettman 1970). By contrast, the importance of other usability categories will be different, depending on product type. For instance, expensive products that are less frequently purchased, such as automobiles-i.e., what Hahn and Kauffman label as "researched goods"promotion is likely to be an important criterion, as better perception of promotion signals quality (Milgrom and Roberts 1986). Likewise, when evaluating intangible goods with differentiation potential, such as car rentals and airlines, consumers will value the emotional aspects of the website, and the madefor-the-medium category that includes personalization will be important. Ease of use is likely to be more important for researched goods because consumers expend considerable effort in the information search activity here. Therefore, we hypothesize as follows.

Hypothesis 4. Product type will have (a) a significant influence on the weights individuals assign to the usability categories of ease of use, promotion, made-for-themedium, and emotion; and (b) a nonsignificant influence on the weights individuals assign to the usability category of content.

Outcomes of Usability. As suggested in the HCI literature, technological artifacts that are more usable are likely to engender greater use. The causal mechanism underlying this relationship is that of motivation: Usable systems not only meet the instrumental goals of users, but also alleviate the cognitive effort associated with use (Nielsen 2000). Further, artifacts that support hedonic goals such as enjoyment lead to greater use (Agarwal and Karahanna 2000). In the context of websites, such use can manifest itself in many different ways: Users may simply visit a website for a brief period of time and move on to another destination in cyberspace, they may spend time browsing a website and exploring its capabilities, or they may visit the website to make a purchase. Regardless of the consumer's goals, we suggest that usability will have a positive effect on use. These theorized effects of usability on use are not only supported by research in HCI but are also consistent with findings in the IS literature. Particularly, the content and ease-of-use categories that are conceptually similar to perceptions of usefulness and ease of use in user acceptance models have consistently explained significant variance in individual technology-use behaviors in a variety of contexts (Venkatesh et al. 2003).

In order to isolate the effects of usability on use behavior, it is important to control for other significant factors that may influence use. Prior technology use, often referred to as habit, has been shown 
to influence future use (Venkatesh et al. 2000), and the role of habit as a determinant of future behavior is established in psychology (e.g., Ouellette and Wood 1998). Habit or routinization is also acknowledged as important in both traditional shopping (Alba and Hutchinson 1987) and online shopping environments (Reibstein 2002). We include prior experience with similar websites and previous product purchases from the website as additional predictors of use behavior. Prior experience, a reflection of a consumer's familiarity with the product, has been shown to strongly influence subsequent behavior (Celci and Olson 1988, Hoch and Deighton 1989). Generally referred to as "learning," the key notion here is that with greater experience, consumers can perform product-related tasks more effectively and have a richer store of prior knowledge to draw upon. Experienced consumers' knowledge structures are more differentiated and hierarchically organized (Hoch and Deighton 1992), and prior product-class knowledge influences both information search behavior (Brucks 1985) and evaluation processes (Sujan 1985). This distinction between experts and novices is echoed in the HCI literature, where researchers have argued and empirically demonstrated that the level of experience and knowledge possessed by a user is an important driver of artifact use (Card et al. 1983). Greater experience with similar sites allows users to develop search and navigational strategies that are more efficient and should, therefore, have a positive effect on use. Likewise, if a consumer has purchased a product previously from a website, there is a greater likelihood of using the website again, simply because of the comfort level and familiarity (Moe and Fader 2004). Therefore, we hypothesize the following.

Hypothesis 5. Individuals' assessment of the content, ease of use, promotion, made-for-the-medium, and emotion categories of usability for a website will predict their use of the website, after controlling for prior experience with similar sites and previous product purchase from the website.

We suggest that use behavior is a determinant of purchase behavior. The amount of time spent in a physical retail outlet such as a mall has been shown to influence the volume of purchases made at a store (e.g., Jarboe and McDaniel 1987). This relationship is not restricted to physical stores alone. It has been argued that the greater the length of time a user spends on a website, the more likely he or she is to purchase the products that are offered on the website (Rosen 2001). Indeed, it is these anticipated benefits of website stickiness that have prompted managers to pay close attention to mechanisms that can help retain visitors at a website. As before, it is important to control for other significant factors that influence consumers' purchase behavior. In predicting purchase behavior, we include two important control variables: (a) purchase need, i.e., the intensity with which the consumer enters the shopping environment with a goal-directed need; and (b) previous product purchase. Purchase behavior is more likely to result when there is a stronger need for the product (Moe 2003), while previous product purchases account for any loyalty the consumer may have developed for the specific store (Huang and $\mathrm{Hu}$ 1999). Therefore, we hypothesize the following.

НүротнеSis 6. Individuals' use of a website will predict purchase behavior on the website, after controlling for purchase need and previous product purchase from the website.

We posit that the goal-oriented and utilitarian categories of usability-i.e., content and made-for-themedium-will exhibit a direct effect on purchase behavior. Whereas the mediated effect via use behavior is likely to be observed for impulse purchases when visitors are merely browsing a website-e.g., in the case of "experiential" shopping (Wolfinbarger and Gilly 2001)—when there is a consumption need that is well specified, a customer may not desire to "linger" on the site, but rather execute a purchase in a timely manner. Wolfinbarger and Gilly (2001) label this type of customer as goal oriented or utilitarian, where accessibility, convenience, and information availability are dominant factors in the consumer's decision process. Thus, the richer the content and personalization offered on the site, the greater the likelihood of a purchase in goal-directed consumer situations. Therefore, we hypothesize as follows.

Hypothesis 7. Individuals' assessment of the content and made-for-the-medium categories of website usability will predict purchase behavior on the website after controlling for website use, purchase need, and previous product purchase from the website.

\section{Method}

\section{Participants}

The population of interest was Internet users. Data were collected in two phases. As reported in Agarwal and Venkatesh (2002), the sampling frame for Phase 1 data collection was visitors to three branches of a major retail store during a three-day period (Friday, Saturday, and Sunday). A total of 920 individuals agreed to participate in the study, and 757 provided usable responses for an effective response rate of $82.3 \%$. Six months later, in Phase 2, we tried to reach all 757 participants, and successfully contacted 384 participants over a two-week period. Of the 384 contacted, 370 of those provided usable responses for an effective response rate of $48.9 \%$ from Phase 1 to Phase 2. Demographic information of the participants 
Table 1 Demographic Characteristics

\begin{tabular}{|c|c|c|c|c|c|c|c|c|}
\hline & \multicolumn{2}{|c|}{ Auto manufacturer } & \multicolumn{2}{|c|}{ Car rental } & \multicolumn{2}{|c|}{ Airline } & \multicolumn{2}{|c|}{ Bookstore } \\
\hline & Phase 1 & Phase 2 & Phase 1 & Phase 2 & Phase 1 & Phase 2 & Phase 1 & Phase 2 \\
\hline$N$ & 201 & 105 & 149 & 69 & 230 & 120 & 177 & 76 \\
\hline$M / F$ & $130 / 71$ & $70 / 35$ & $95 / 54$ & $41 / 28$ & $151 / 79$ & $81 / 39$ & $111 / 66$ & $50 / 26$ \\
\hline Age & $31.07(6.84)$ & $30.77(6.91)$ & $30.98(6.80)$ & $32.10(7.01)$ & $30.07(6.62)$ & $31.03(6.89)$ & $30.01(6.64)$ & $31.34(6.71)$ \\
\hline Income & $57,987(7,922)$ & $56,217(7,158)$ & $56,545(7,887)$ & $55,420(7,712)$ & $57,004(8,275)$ & $54,132(7,988)$ & $58,145(8,045)$ & $56,130(8,132)$ \\
\hline
\end{tabular}

Note. Mean and standard deviation (in parentheses) pairs are indicated for age and income.

in both phases, broken down by the various industries, is shown in Table 1. A comparison confirms comparable demographics across both phases.

\section{Procedure and Measurement}

The procedure for Phase 1 is described in detail in Agarwal and Venkatesh (2002) and summarized below. Participants were recruited at three branch locations of a major electronics retail store. Three kiosks each were set up in each of the three locations of the store for participants to browse the specific websites being studied and also respond to the questionnaire. The use of three kiosks at each store helped minimize participant wait time. As noted earlier, websites were chosen from four industries, with five or six companies being chosen in each industry-auto manufacturers (BMW, Chrysler, Ford, GM, Mercedes, and Porsche), car rental (Alamo, Avis, Budget, Hertz, and National), airlines (American, Delta, Northwest, United, and US Airways), and bookstores (Amazon, Barnes and Noble, Booksense, Borders, and Varsity Books). ${ }^{1}$

As discussed earlier, four types of products were examined here: (a) automobiles, representing high outlay, infrequently purchased, tangible value proposition, and high differentiation potential in the Peterson et al. (1997) classification, (b) car rentals and airline reservations, representing moderate outlay, purchased somewhat frequently, intangible value proposition, and moderate differentiation potential, and (c) books, representing low outlay, frequently purchased, tangible value proposition, and low differentiation potential. The selection of websites was purposively made to include websites that differ in their specific characteristics (e.g., design features), thereby ensuring variance in ratings. For instance, in bookstores, we included a leader (e.g., barnesandnoble.com) and a no-frills store (e.g., booksense.com). Similarly, car rentals included companies targeting varying types of customers, such as Budget, Alamo, and Hertz. Only in airlines did we specifically not include an airline such

\footnotetext{
${ }^{1}$ In order to protect the anonymity of these sites and the organizations they represent, sites are listed here alphabetically and do not represent the order in which they were entered in the data file.
}

as Southwest. The rationale for doing so was because we wanted to ensure that most customers would have had an opportunity to deal with one or more of the airlines, and at the time of the study, Southwest was not a major carrier in the geographic region where this study was conducted.

When a participant arrived at a kiosk, he or she was prompted with a request to fill out a survey as a customer of websites in one of the four industries, with the specific industry being randomly selected by the computer. $^{2}$ The measurement of usability using this instrument was a multistep process. First, users assign weights to each of the five categories by distributing 100 points to reflect their assessments of each category's importance in determining the usability of all websites that offer a specific product. Thus, the usability category weights governed all the websites that a participant was evaluating. Following this, the participants visited the websites, with the order of presentation of the different websites being randomized by the computer. Every participant was given five or more minutes to browse each website. After browsing each website, the participants rated the site on the different MUG subcategories on a 10-point scale. The product of the weights and ratings together, aggregated across all subcategories in a category, provided an individual's weighted rating for each usability category. Finally, information regarding the participant's prior experience with similar websites, purchase need related to the product type, previous purchase on the websites, and demographic characteristics was collected. ${ }^{3}$ More details about the metric and the

\footnotetext{
${ }^{2}$ The data were gathered from 1,475 individuals in Phase 1, performing either the customer or investor task (see Agarwal and Venkatesh 2002). However, for the purpose of this paper, only the data related to the customer are being used.

${ }^{3}$ We also collected data on individuals' prior Internet experience, prior experience with the specific websites, and computer self-efficacy as additional controls because of findings from prior research that these variables are related to technology use (e.g., Venkatesh et al. 2003). They were not significantly related to the outcomes examined here and, hence, these variables were not included. The nonsignificance of these variables is likely due to their effects being captured by other constructs in the model. For example, self-efficacy was likely reflected in ease-of-use
} 
associated measurement procedure are available in Agarwal and Venkatesh (2002).

Phase 2 was a telephone interview of participants from Phase 1. The interviews were conducted by individuals in the telemarketing department at the sponsor firm. They possessed about six months of experience in telephone-based services. The objective of Phase 2 was to collect behavioral data for the six months subsequent to Phase 1 . Up to 30 callbacks were attempted to reach the participants in Phase 2. The average phone call lasted less than four minutes. During the phone interview, participants were asked about their use of the various websites that they had rated in Phase 1, and their purchases from the websites. The items, other than those measuring usability, which are reported in Agarwal and Venkatesh (2002), are shown in the appendix (available online at http:// mansci.pubs.informs.org/ecompanion.html). Website use was measured using four formative indicatorsfrequency of use (first item), duration of use (second and third items), and intensity of use (fourth item) over the past six months. Purchase behavior was measured using two formative indicators: frequency and amount of purchase in the past six months.

\section{Results}

We analyzed the data using the structural equation modeling technique of partial least squares (PLS). The software used for the analysis was PLS Graph, Version 2.91.03.04. PLS is effective to test a causal model such as the one proposed in Figure 1. Two reasons for the applicability of PLS here are (1) PLS does not make distributional assumptions, unlike LISREL, and is therefore suitable for situations where the data might violate normality assumptions (Chin 1998); and (2) PLS allows the modeling of constructs with formative indicators.

PLS estimates a measurement model for each structural model tested. The measurement model provides information about the reliability and validity of various scales, particularly multi-item scales using reflective indicators. In the models tested here, some constructs were operationalized using single items (e.g., gender, age, income) and others were modeled using formative indicators (e.g., use behavior, purchase behavior), thus reliability and validity statistics were not applicable in all cases. In cases where reliability and validity were applicable (e.g., purchase need), high reliability and validity were found based on internal consistency reliability (ICR) and average variance extracted (AVE). For analytical purposes, product characteristics were captured

perceptions, while Internet experience was likely more distal from use behavior than prior experience with similar sites. via three dummy variables-Product1, Product2, and Product3 - that were coded as a $0 / 0 / 0$ for the airlines, $1 / 0 / 0$ for the bookstores, $0 / 1 / 0$ for auto manufacturers, and $0 / 0 / 1$ for the car rental industry. Further, in order to test the model, the interaction terms were created at the level of the indicator per the guidelines of Chin et al. (1996). ${ }^{4}$

Descriptive statistics and correlations for the Phase 2 data are shown in Table 2. Purchase was most highly correlated with use. Use, in turn, was correlated with the different weighted ratings-highest with content, ease of use, and made-for-the-medium. For the other constructs in the model, gender and age are correlated with many of the variables, with the correlations with the product dummy variables being about 0.20 or greater. The pattern of correlations between gender, age, and the various weights are in the hypothesized direction.

We tested two different models using the Phase 2 $(N=370)$ data. ${ }^{5}$ The first model examined the specific effects of demographic characteristics and product characteristics on the weights placed on each category. In the second model, using category-level weighted ratings and control variables as predictors, we examined use behavior as the dependent variable. Also in this model, utilizing use behavior, category-level weighted ratings, purchase need, and previous purchase as predictors, we examined purchase behavior as the dependent variable. Time spent browsing each site was used as a control variable but was found to be nonsignificant in all cases and, therefore, dropped, and the models were reestimated. The other control variables related to experience did not have an effect on both behaviors and were also dropped and the models reestimated, which is standard practice in model estimation to eliminate shared variance between significant and nonsignificant constructs, thus permitting a more accurate understanding of the predictive ability of the significant constructs.

The models for weights were reasonably well supported, with $R^{2}$ ranging from $21 \%$ to $43 \%$. Table 3 presents the results. Gender influenced the weights of four of the five categories, with content and made-forthe-medium being more important to men, and ease of use and emotion being more important to women, but the weight on promotion did not differentiate on

\footnotetext{
${ }^{4}$ If $\mathrm{A} \times \mathrm{B}$ is being modeled and $\mathrm{A} 1, \mathrm{~A} 2$, and $\mathrm{A} 3$ are the indicators of the construct $\mathrm{A}$, and $\mathrm{B} 1$ and $\mathrm{B} 2$ are the indicators of the construct $\mathrm{B}$, the latent variable $A \times B$ is created with the following indicatorlevel product terms: $\mathrm{A} 1 \times \mathrm{B} 1, \mathrm{~A} 1 \times \mathrm{B} 2, \mathrm{~A} 2 \times \mathrm{B} 1, \mathrm{~A} 2 \times \mathrm{B} 2, \mathrm{~A} 3 \times \mathrm{B} 1$, and $\mathrm{A} 3 \times \mathrm{B} 2$.

${ }^{5}$ We also tested the left-hand side of the model-i.e., the effects of individual characteristics and product type-on the sample from Phase $1(N=757)$. The pattern of results from the two different sets of tests was identical.
} 

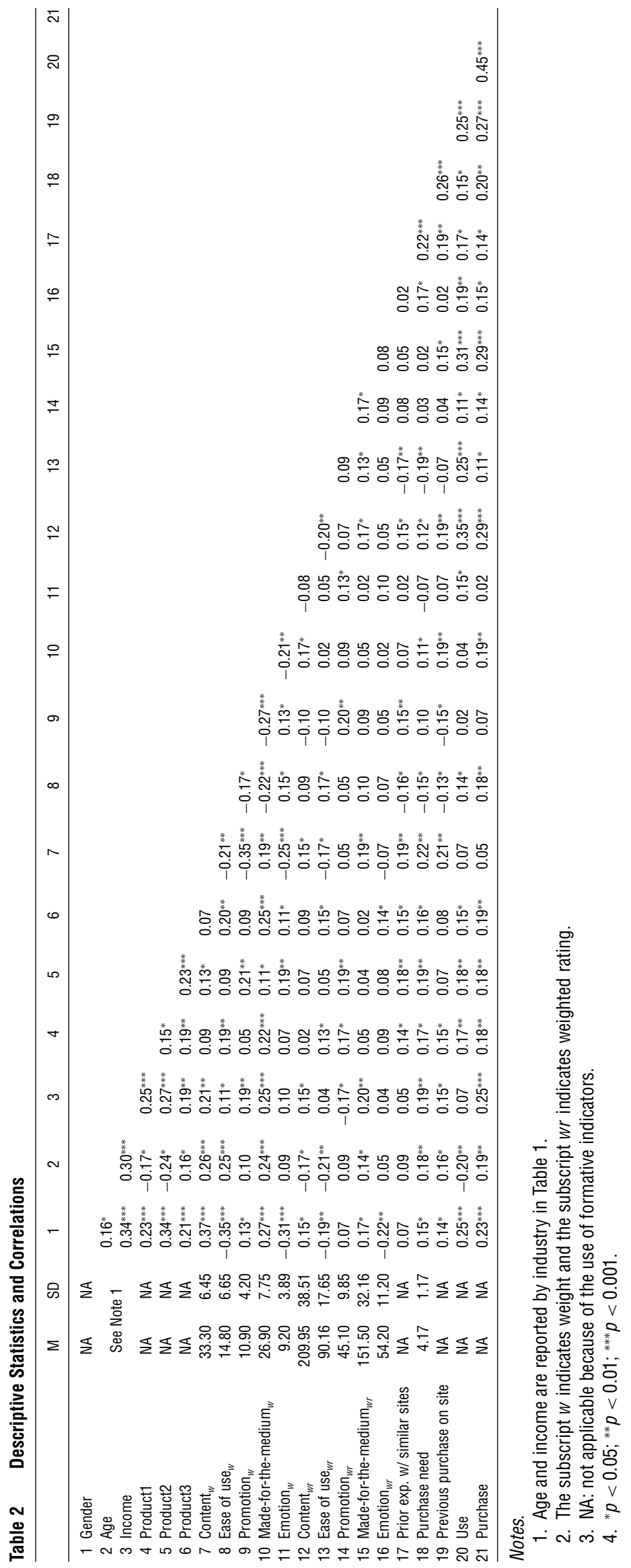
Table 3 Predicting Category Weights

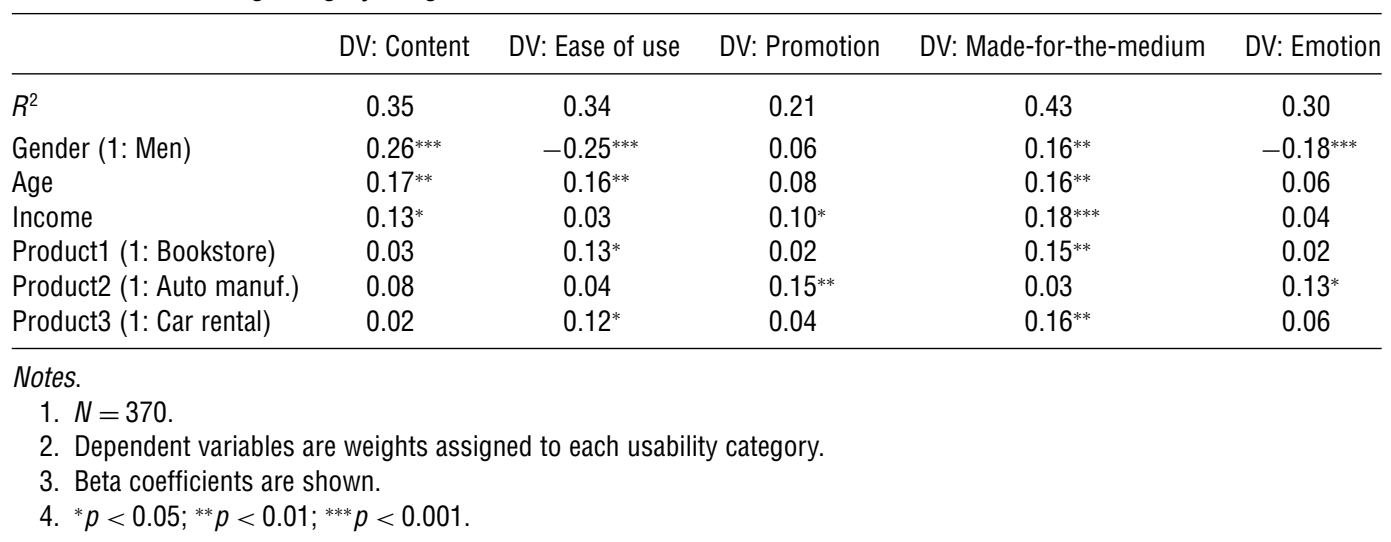

the basis of gender. This provides partial support for Hypothesis 1. While the hypothesis related to greater importance of promotion to women was not supported, in addition, made-for-the-medium being more important to men was contrary to expectations that there would be no difference between genders. Perhaps made-for-the-medium was driven more by the content and task-oriented objectives than the efficiency objectives, thus making it more important to men when compared to women.

Age influenced the weights placed on content, ease of use, and made-for-the-medium, with all three categories being more important to older people; no effects were observed on promotion or emotion. Hypothesis 2 was, thus, mostly supported. The only contrary finding was that made-for-the-medium was more important to older consumers and not to younger consumers. As was the case of gender, the possible reason for this is the emphasis on the content, task, and effectiveness aspects of madefor-the-medium, which are more important to older consumers, rather than any of the other aspects such as e-community or uniqueness that would be more important to younger consumers.

Income influenced the weights placed on content, promotion, and made-for-the-medium, with all three categories being more important to higher-income consumers; ease of use and emotion were not differentiated on the basis of income. Thus, Hypothesis 3 was mostly supported. The only contrary finding here was that ease of use was not differentiated by income, in contrast to our expectation that it would be more important to higher-income consumers because they would be time-starved. Often, higher levels of income are associated with higher levels of education, possibly resulting in higher levels of technology competence, which is generally accompanied by a reduced importance of ease of use (see Venkatesh et al. 2003). Thus, such high levels of technology competence may have counteracted any effects due to time starvation.
Using the three categorical variables to operationalize product type, we found that the weights placed on different usability categories did, in fact, discriminate on the basis of product type. Specifically, with the exception of content, the weights placed on the other four categories were influenced by the product dummy variables, thus supporting Hypothesis 4(a) and Hypothesis 4(b).

Use was predicted by a combination of weighted ratings of MUG categories, demographic characteristics, and previous product purchase. Table 4 presents the results. Once again, most of the factors suggested in Hypothesis 5 did indeed predict use. Content, made-for-the-medium, and ease of use were the most important (in order of importance) predictors of use. Previous product purchase was the next most important predictor, while gender (greater use among men) and age (greater use among younger people) were also significant, but income was not significant. Also, emotion had a small but significant effect on use, despite its low weights (see Agarwal

Table 4 Predicting Use and Purchase

\begin{tabular}{lcc}
\hline & DV: Use & DV: Purchase \\
\hline$R^{2}$ & 0.53 & 0.53 \\
Use & - & $0.30^{* * *}$ \\
Usability scores (Weight $*$ Rating) & $0.32^{* * *}$ & $0.22^{* * *}$ \\
$\quad$ Content & $0.21^{* *}$ & 0.02 \\
$\quad$ Ease of use & 0.08 & 0.07 \\
$\quad$ Promotion & $0.29^{* * *}$ & $0.21^{* * *}$ \\
$\quad$ Made-for-the-medium & $0.13^{*}$ & 0.08 \\
$\quad$ Emotion & 0.07 & - \\
Prior experience with similar sites & - & $0.13^{*}$ \\
Purchase need & $0.19^{* *}$ & $0.20^{* *}$ \\
Previous product purchase on website & & \\
\hline Notes. & & \\
1. $N=370$. & \\
2. Cells with a long dash are not applicable in the context of a specific \\
model. \\
3. Beta coefficients are shown. \\
$\quad$ 4. ${ }^{*} p<0.05 ;{ }^{* *} p<0.01 ;{ }^{* * *} p<0.001$.
\end{tabular}


and Venkatesh 2002), while promotion, another lowweighted category, did not have any effect. Perhaps prior experience with the sites was not significant because it likely shared variance with previous product purchase on the website. Thus, overall, the MUG categories demonstrated a high degree of predictive validity on use behavior measured over a six-month period, with an explained variance of $53 \%$.

Similar to use, purchase behavior was predicted by a combination of factors. The proposed partially mediated relationships presented in Hypotheses 6-7 were supported (see Table 4). Use had the strongest effect on purchase, while weighted ratings of content and made-for-the-medium had a direct effect on purchase beyond what was mediated by use. This is consistent with our theory that quality content, including tailored content, can in fact result in purchase without the need for high levels of site use. As expected, both control variables-purchase need and previous purchase-had a significant effect on purchase behavior. ${ }^{6}$ Collectively, the proposed predictors accounted for $53 \%$ of the variance in purchase behavior, thus providing a good explanation of the key dependent variable.

\section{Discussion}

\section{Summary of Findings and Limitations}

The goal of this paper was to offer a usability-centric perspective on purchase behavior in electronic channels by developing a nomological net for website usability. Drawing on IS and HCI research, we argued that the usability of a website would influence both the extent to which the website is able to attract and retain visitors, as well as the transformation of these visitors into actual consumers. Based on HCI research that suggests that usability is a subjective construct, we used consumers' perceived usability as a central construct in our model, in contrast to much prior work where the researchers assessed usability (e.g., Lohse and Spiller 1999).

We further described a range of individual and product factors that are influential determinants of the importance consumers place on various usability categories. Empirical results provided support for a majority of the proposed relationships and the critical role of perceived usability in our model. Additionally, the longitudinal nature of the study supports the theorized existence of the causal relationship between usability and use behavior, and usability and purchase behavior. The inclusion of relevant control variables such as prior experience with similar sites,

\footnotetext{
${ }^{6}$ Although not shown, we also tested a model that included two interaction terms-use and purchase need, and use and previous purchase-and found both interaction terms to be nonsignificant.
}

previous purchase behavior on the websites, and purchase need in examining use and purchase behaviors allowed us to isolate the effects of usability on these key outcomes. Finally, the large sample size and the fact that we examined multiple websites from diverse industries enhance the external validity of our results.

Prior to discussing the implications of our findings, however, it is important to acknowledge the limitations of this research. We used self-report measures of actual purchase behavior rather than objective data. Nonetheless, because respondents in Phase 2 of the study had no incentive to misrepresent their frequency and volume of purchases, we do not view this as a major limitation. The data for use behavior and purchase behavior were gathered at the same point in time; therefore, although causality is theorized, it cannot be established beyond reasonable doubt in this study. Some nonsignificant findings should be interpreted with caution until replicated because of the potential for type II error. For instance, demand factors of the experimental setting may have suppressed the importance that participants assign to emotion. Similarly, the importance of promotion may have been reduced because participants did not have an opportunity to enter the specific website from another site where it was being "promoted."

Additionally, as noted in Agarwal and Venkatesh (2002), although the mall-intercept technique is an accepted research design for studying consumer populations (e.g., Cowan 1989), the sampling method can pose a potential threat in regard to selection bias. However, the fact that data were collected over three days in three different locations mitigates this concern somewhat. Based on our sampling technique, the generalizability of the findings is limited to consumer populations that typically shop in malls and to the types of products we studied. Finally, we were unable to establish contact with all of the respondents from Phase 1 to obtain follow-up data in Phase 2. Nonetheless, we were able to obtain a sample size of over 350 to test the full research model.

\section{Implications for Research}

IS researchers have long sought to characterize the success of an information system using a variety of outcomes ranging from information satisfaction, to user satisfaction, and to actual use (DeLone and McLean 1992). However, much of the research on technology acceptance and use stops at the prediction of use behavior, where the link between use and performance outcomes is implied but not tested (e.g., Venkatesh and Davis 2000). Indeed, there has been limited research that integrates system success measures with outcomes of organizational interest such as purchases. An exception worth noting here is 
the recent work of Devaraj and Kohli (2003), where, using longitudinal data from hospitals, technology investments were related to outcomes such as hospital revenue and quality, with technology use as a key mediator. In a similar spirit, our study suggests that in the context of B2C electronic commerce, use behavior and usability exhibit important downstream effects on sales. Future research is necessary to study the relationship between actual use of websites and other firm-level outcomes.

We described a nomological network for usability that can serve as the foundation for future work. Drawing upon research in marketing, we proposed two sets of drivers of the importance of usability categories. Future research can build on literature from other disciplines such as psychology to extend the nomological net and incorporate additional determinants of usability such as relatively stable personality characteristics (Digman 1990) or more transient "state" variables such as mood (Hill and Ward 1989). We did not include task in our model. A logical extension to this work, therefore, would be to extend the model to incorporate task types such as the distinction made by Wolfinbarger and Gilly (2001) between "experiential" and "goal-directed" shopping, or by Moe (2003) between directed buying, search/deliberation, hedonic browsing, and knowledge building. In particular, our focus here has been on business-oriented sites, whereas more entertainment-oriented sites could cause customers to greatly vary their weighting scheme because of the nature of the interactions. For instance, it could be argued that because entertainment sites typically serve hedonic goals, among the usability categories, emotion will dominate in importance for users (see Agarwal and Karahanna 2000).

Likewise, although we examined "products" from four different industries, there are many flavors of B2C sites that support different types of transactions. Thus, for example, this work could be replicated for sites that are purely content oriented or that offer online service capabilities to determine if the findings generalize. Such work would lead to a more comprehensive view of the role of usability and also help us identify potential contingencies tied to particular types of websites. Further, our use of categorical variables for product type and the finding related to their significant effects suggests the need to refine our theoretical understanding of these relationships by proposing more specific relationships between different types of products and the importance of usability categories. For such theoretical development, products could be differentiated on the basis of a number of taxonomies. In our study, we selected product categories based on our assessment of the specific characteristics identified by Peterson et al. (1997), such as frequency of purchase, differentiation potential, and outlay. As discussed, Hahn and Kauffman (2002) provided a different product taxonomy that could be used to further deepen our understanding of the role of the product in driving usability. In future research, it would also be useful to directly measure these specific product characteristics using objective or perceptual data. Although we identified the drivers of category weights, our theorizing did not include predictions about the drivers of ratings. Future research should study the predictors of ratings by including variables such as specific website design features and individual preferences to further extend the nomological network.

Overall, our work complements and extends current thinking in $\mathrm{HCI}$, IS, and consumer behavior research on the role of perceived usability as a critical predictor of use behavior and purchase behavior. In our conceptualization of the determinants of purchase behavior, we presented a complementary view to the traditional belief-attitude-behavior models that have been dominant in IS research (e.g., Taylor and Todd 1995, Venkatesh and Davis 2000). An interesting extension would be to examine the relative explanatory power of a usability-based model versus a social psychology model. Additional research is necessary to integrate these perspectives into a more holistic model. Given the maturity of technology adoption research, this "new" usability-centric view may yield novel theoretical and empirical insights that will provide a basis for furthering our understanding of technology use. Related to this, it will be useful to see if the usability characteristics are possible determinants of overall technology perceptions such as those described in technology acceptance theories (see Venkatesh et al. 2003). Future research could also extend the proposed model to include other determinants of purchase behavior, such as perceptions of the sales process and Internet search to isolate the additional variance explained by usability and use behavior.

The nomological network presented here is important and provides an integrated view of a firm's Web presence and its impact, but the model is primarily a predictive one. Future research should study more malleable constructs that may be the target for design, training, and other managerial interventions. Additionally, our investigation and conceptualization of usability has treated it as a subjective construct, consistent with a large body of work in IS and HCI. However, alternative conceptualizations that view usability as a more objective construct using, say, a keystroke model (e.g., Card et al. 1983, Jeffries et al. 1991) should also be considered, particularly for a comparison to the results from the current work. Overall, the relationships tested here represent 
an important initial step toward a model that serves as critical input for website design in a wide variety of person-situation configurations.

One of the strengths of the current work is the use of a longitudinal data set from consumers, but such field settings come with some obvious limitations related to potential confounding factors. An important future research direction that stems from this is the need to conduct experimental studies. In addition to enhancing internal validity, such studies can be used as a test bed to examine the effectiveness of various design interventions that are informed by the results of the current work. In particular, experimental research would be useful in determining the drivers of ratings and their relative impacts on user evaluations.

\section{Implications for Practice}

Our findings have important implications for firms that desire to establish a successful Web presence. Assuming that one measure of online success is the volume of purchases made at a website, our results indicated that the website usability explained significant variance in purchase behavior via a direct path, after controlling for other important drivers of purchase behavior. Clearly then, the first managerial recommendation that emerges is the importance of paying close attention to the usability of a website that is to be used by customers for transaction purposes. The fact that usability also influences purchase behavior via a path only partially mediated by use further underscores its significance. Usable websites cause visitors to remain on the site for extended periods of time, thereby increasing the likelihood of an ultimate purchase. As demonstrated in the findings of Rozanski et al. (2001), "loiterers" and "surfers" have among the lengthiest online sessions during a typical interaction on the Web and also, reported the highest levels of purchasing behavior. Our findings reinforce the importance of usability testing for organizations, with one mechanism for performing such testing being collaborations between researchers and practitioners.

At a more granular level, important managerial insights are embedded in the specific relationships that we found between individual and product factors and the weights assigned to usability categories. For instance, our results show that men value content and personalization on a website, while women value ease of use and emotion. Practitioners can use these findings to design websites that specifically match the requirements of different consumer populations based on individual characteristics. Our results also show that critical categories of usability are influenced by the nature of the product the website is offering. Not surprisingly, content is uniformly viewed as important across all product types, underscoring other work that has characterized the Internet as a channel that significantly enhances information availability (e.g., Bakos 1997). Additionally, product type influenced the weights assigned to other usability categories-for instance, ease of use is perceived by consumers to be more important for bookstores and online car rentals, as compared with airlines; and there is no significant difference in the importance of ease of use between auto manufacturers and airlines. Although specific recommendations are possible only with additional studies, these findings nevertheless have important implications for resource allocation in website design. Depending on the nature of the product that is being sold on the website, managers should seek to invest the most in those categories of website usability that consumers rate as being most important.

\section{Conclusions}

As indicated by recent data comparing overall retail sales to sales via electronic channels (Census Bureau 2003), it is evident that B2C e-commerce represents a huge market opportunity for firms seeking to expand their touch points with customers. A successful Web presence that yields actual sales requires an understanding of both IS, HCI, and marketing. This research, situated at the nexus of IS, HCI, and marketing, represents one effort in that direction. In this work, we empirically demonstrated that a rich and detailed conceptualization of website usability could have a strong effect on sales, and thus, a firm's bottom line. The model presented here-i.e., including individual characteristics, product type, use behavior, and purchase behavior-provides the basis for further inquiry to include experimental studies, moderating influences, and the addition of other variables. As organizations continue to expand their Web presence, the drivers of usability identified here can assist managers in formulating appropriate website design and redesign strategies.

An online supplement to this paper is available on the Management Science website (http://mansci.pubs. informs.org/ecompanion.html).

\section{References}

Agarwal, R., E. Karahanna. 2000. Time flies when you're having fun: Cognitive absorption and beliefs about information technology use. MIS Quart. 24(4) 665-694.

Agarwal, R., J. Prasad. 1998. A conceptual and operational definition of personal innovativeness in the domain of information technology. Inform. Systems Res. 9(2) 204-215.

Agarwal, R., V. Venkatesh. 2002. Assessing a firm's Web presence: A heuristic evaluation procedure for the measurement of usability. Inform. Systems Res. 13(2) 168-186. 
Agarwal, R., P. De, A. Sinha. 1999. Comprehending object and process models: An empirical study. IEEE Trans. Software Engrg. 25 541-556.

Alba, J. W., J. W. Hutchinson. 1987. Dimensions of consumer expertise. J. Consumer Res. 13 411-454.

Bakos, Y. 1997. Reducing buyer search costs: Implications for electronic marketplaces. Management Sci. 43(12) 1676-1692.

Bao, Y., A. T. Shao. 2002. Nonconformity advertising to teens. J. Advertising Res. 42(3) 56-65.

Barfield, W., T. A. Furness, eds. 1997. Virtual Environments and Advanced Interface Design. Oxford University Press, New York.

Barletta, M. 2003. Marketing to Women: How to Understand, Reach, and Increase Your Share of the World's Largest Market. Dearborn Trade Publications, Chicago, IL.

Belk, R. W., M. Wallendorf, J. F. Sherry, Jr. 1989. The sacred and the profane in consumer research. J. Consumer Res. 16 1-38.

Bettman, J. R. 1970. Information processing models of consumer behavior. J. Marketing Res. 7 370-376.

Brucks, M. 1985. The effects of product class knowledge on information search behavior. J. Consumer Res. 12 1-16.

Burke, R. R. 2002. Technology and the customer interface: What consumers want in the physical and virtual store. J. Acad. Marketing Sci. 30(4) 411-432.

Burroughs, R. E., R. Sabherwal. 2002. Determinants of retail electronic purchasing: A multi-period investigation. Infor 40(1) $35-56$.

Card, S. K., T. P. Moran, A. Newell. 1983. The Psychology of HumanComputer Interaction. Erlbaum, Hillsdale, NJ.

Celci, R. L., J. C. Olson. 1988. The role of involvement in attention and comprehension processes. J. Consumer Res. 15 210-224.

Census Bureau. 2003. Retail 4Q: 2002 Ecommerce report. http:// www.census.gov/mrts/www/current.html.

Chen, L.-D., M. L. Gillenson, D. L. Sherrel. 2002. Enticing online consumers: An extended technology acceptance perspective. Inform. Management 39(8) 705-719.

Chiang, K.-P., R. T. Dholakia. 2003. Factors driving consumer intention to shop online: An empirical investigation. J. Consumer Psych. 13(1-2) 177-183.

Chin, W. W. 1998. Issues and opinion on structural equation modeling. MIS Quart. 22 vii-xvi.

Chin, W. W., B. L. Marcolin, P. R. Newsted. 1996. A partial least squares latent variable modeling approach for measuring interaction effects: Results from a Monte Carlo simulation study and voice mail emotion/adoption study. J. I. DeGross, S. L. Jarvenpaa, A. Srinivasan, eds. Proc. Internat. Conf. Inform. Systems, AIS, Cleveland, OH, 21-41.

Cowan, C. 1989. Mall intercepts and clinical trials: The philosophy of inference from different types of research designs. Marketing Res. 1(1) 15-22.

Dawar, N., S. Ratneshwar, A. G. Sawyer. 1992. The use of multiple methods to explore three-way person, brand and usage context interactions. Adv. Consumer Res. 19 116-122.

DeLone, W. H., E. R. McLean. 1992. Information systems success: The quest for the dependent variable. Inform. Systems Res. 3(1) 60-95.

Devaraj, S., R. Kohli. 2003. Performance impacts of information technology: Is actual usage the missing link? Management Sci. 49(3) 273-289.

Diehl, K., L. J. Kornish, J. G. Lynch. 2003. Smart agents: When lower search costs for quality information increases price sensitivity. J. Consumer Res. 30 56-71.

Digman, J. M. 1990. Personality structure: Emergence of the fivefactor model. Ann. Rev. Psych. 41 417-440.

Eighmey, J., L. McCord. 1998. Adding value in the information age: Uses and gratifications of sites on the World-Wide Web. J. Bus. Res. 41 187-194.
Fay, W. B. 1993. Understanding generation X. Marketing Res. 5 $54-55$.

Feller, B. 2003. Students rule net population. Marketing News 37(24),

Hahn, J., R. J. Kaufman. 2002. Information foraging in Internetbased selling: A systems design value assessment framework. M. Shaw, ed. E-Business Management: Integration of Web Technologies with Business Models. Kluwer Academic Publishers, New York, 195-230.

Haubl, G., V. Trifts. 2000. Consumer decision making in online shopping environments: The effects of interactive decision aids. Marketing Sci. 19(1) 4-21.

Hawkins, D. I., R. J. Best, K. A. Coney. 1995. Consumer Behavior: Implications for Marketing Strategy. Richard D. Irwin, Chicago, IL.

Hill, R. P., J. C. Ward. 1989. Mood manipulation in marketing research: An examination of potential confounding effects. J. Marketing Res. 26(1) 97-104.

Hoch, S. J., J. Deighton. 1989. Managing what consumers learn from experience. J. Marketing 53(2, 3) 1-20.

Huang, M.-H., S. Yu. 1999. Are consumers inherently or situationally brand loyal? A set intercorrelation account for conscious brand loyalty and nonconscious inertia. Psych. Marketing 16(6) 523-544.

Jarboe, G. R., C. D. McDaniel. 1987. A profile of browsers in regional shopping malls. Acad. Marketing Sci. J. 15(1) 46-63.

Jarvenpaa, S. L., P. A. Todd. 1996/1997. Consumer reactions to electronic shopping on the World Wide Web. Internat. J. Electronic Commerce 1(2) 59-88.

Jeffries, R. J., J. R. Miller, C. Wharton, K. M. Uyeda. 1991. User interface evaluation in the real world: A comparison of four techniques. Proc. CHI Human Factors Comput. Systems. ACM, New Orleans, Los Angeles, New York, 119-124.

Karat, J. 1997. Evolving the scope of user-centered design. Comm. ACM 40 33-38.

Lais, S. 2002. How to stop Web shopper flight. Computerworld (June 17) 44-45.

Lecerof, A., F. Paterno. 1998. Automatic support for usability evaluation. IEEE Trans. Software Engrg. 24 863-887.

Lohse, G., P. Spiller. 1999. Internet retail store design: How the user interface influences traffic and sales. J. Comput.-Mediated Comm. 5.

Lynch, J. G., D. Ariely. 2000. Wine online: Search cost affect competition on price, quality, and distribution. Marketing Sci. 19(1) 83-103.

Milewski, A. E., T. M. Smith. 2000. Providing presence cues to telephone users. Proc. Computer Supported Cooperative Work. ACM Press, New York, 89-96.

Milgrom, P. R., J. Roberts. 1986. Price and advertising signals of product quality. J. Political Econom. 94 796-821.

Moe, W. W. 2003. Buying, searching, or browsing: Differentiating between online shoppers using in-store navigational clickstream data. J. Consumer Psych. 13(1-2) 29-39.

Moe, W. W., P. S. Fader. 2004. Dynamic conversion behavior at e-commerce sites. Management Sci. 50(3) 326-335.

Morton, F. S., F. Zettlemeyer, J. Silva-Risso. 2001. Internet car retailing. J. Indust. Econom. 49(4) 501-519.

Moschis, G. P. 1991. Marketing to older adults: An overview and assessment of current knowledge and practice. J. Consumer Marketing 8(3) 33-41.

Nielsen, J. 2000. Designing Web Usability. New Riders, Indianapolis, IN.

Olson, G. M., J. S. Olson. 2003. Human computer interaction: Psychological aspects of the human use of computing. Ann. Rev. Psych. 54 491-516. 
Ouellette, J. A., W. Wood. 1998. Habit and intention in everyday life: The multiple processes by which past behavior predicts future behavior. Psych. Bull. 124(1) 54-74.

Palmer, J. W. 2002. Web site usability, design, and performance metrics. Inform. Systems Res. 13(2) 151-167.

Pashigian, B. P., S. Peltzman, J.-M. Sun. 2003. Firm responses to income inequality and the cost of time. Rev. Indust. Organ. 22(4) 253-273.

Peterson, R. A., S. Balasubramaniam, B. J. Bronnenberg. 1997. Exploring the implications of the Internet for consumer marketing. J. Acad. Marketing Sci. 25 329-346.

Prakash, V. 1992. Sex roles and advertising preferences. J. Advertising Res. 32(3) 43-52.

Reibstein, D. J. 2002. What attracts customers to online stores, and what keeps them coming back? Acad. Marketing Sci. J. 30(4) 465-473.

Rosen, S. 2001. Sticky website is key to success. Comm. World 36, 36.

Rozanski, H. D., G. Bollman, M. Lipman. 2001. Seize the occasion: Usage based segmentation for Internet marketers. E Insights Booz Allen and Hamilton, McLean, VA.

Seddon, P. B. 1997. A respecification and extension of the DeLone and McLean model of IS success. Inform. Systems Res. 8(3) 240-254.

Shneiderman, B. 1980. Software Psychology: Human Factors in Computer and Information Systems. Winthrop, Cambridge, MA.

Simmons Market Research Bureau. 1991. Study of Media and Markets. Simmons Market Research Bureau, New York.

Smith, S. M., D. B. Whitlark. 2001. Men and women online: What makes them click? Marketing Res. 13(2) 20-25.

Sujan, M. 1985. Consumer knowledge: Effects on evaluation strategies mediating consumer judgments. J. Consumer Res. 12 31-46.
Taylor, S., P. A. Todd. 1995. Understanding information technology usage: A test of competing models. Inform. Systems Res. 6 144-176.

Triplett, T. 1994. Automaker's recognizing value of women's markets. Marketing News 28(8) 1-2.

Vakratsas, D. 1998. Household cost effects on purchase timing decisions: Do demographics matter? J. Consumer Marketing 15(1) 6-22.

Van Slyke, C., C. L. Comunale, F. Belanger. 2002. Gender differences in perceptions of Web-based shopping. Comm. ACM 45(8) $82-86$.

Venkatesh, V. 2000. Determinants of perceived ease of use: Integrating perceived behavioral control, computer anxiety and enjoyment into the technology acceptance model. Inform. Systems Res. 11 342-365.

Venkatesh, V., F. D. Davis. 2000. A theoretical extension of the technology acceptance model: Four longitudinal field studies. Management Sci. 46 186-204.

Venkatesh, V., M. G. Morris. 2000. Why don't men ever stop to ask for directions? Gender, social influence, and their role in technology acceptance and usage behavior. MIS Quart. 24(1) 115-139.

Venkatesh, V., M. G. Morris, P. L. Ackerman. 2000. A longitudinal field investigation of gender differences in individual technology adoption decision-making processes. Organ. Behavior Human Decision Processes 83(1) 33-60.

Venkatesh, V., M. G. Morris, G. B. Davis, F. D. Davis. 2003. User acceptance of information technology: Toward a unified view. MIS Quart. 27(3) 425-478.

Wolfinbarger, M., M. C. Gilly. 2001. Shopping online for freedom, control, and fun. California Management Rev. 32(2) 34-55.

Zinn, L. 1991. This Bud's for you. Business Week (November 4) 90. 